\title{
An Alport syndrome boy with Van Wyk-Grumbach syndrome induced by prolonged untreated congenital hypothyroidism
}

\author{
Su-Jeong Lee, MD', \\ Jung-Eun Moon, $\mathrm{MD}^{1}$, \\ Gi-Min Lee, MD', \\ Min-Hyun Cho, MD², \\ Cheol Woo Ko, MD, PhD ${ }^{1}$ \\ 'Division of Pediatric Endocrinology, \\ Kyungpook National University Child- \\ ren's Hospital, Daegu, Korea \\ 2Division of Pediatric Nephrology, \\ Kyungpook National University Child- \\ ren's Hospital, Daegu, Korea
}

\begin{abstract}
Alport syndrome (AS) is a rare genetic disorder that causes progressive nephritis and is more common among males. Studies have reported an association between thyroid antibodies and hypothyroidism in patients with AS, but the relevance of this relationship is under debate. Prolonged untreated hypothyroidism induces short stature, abnormal pubertal development, and various other symptoms. However, children with long-standing hypothyroidism rarely present with signs of precocious puberty, or Van Wyk-Grumbach syndrome (VWGS). We report the case of a boy, 8 years and 4 months old, who had VWGS caused by prolonged untreated congenital hypothyroidism and AS. The boy had repeated gross hematuria and proteinuria and was diagnosed with AS by renal biopsy and genetic testing. He had normal renal function but severe growth retardation and hypothyroidism. Obesity, bone age delay, hyperlipidemia, and abnormal increased testicle size were also present due to prolonged untreated hypothyroidism. His thyroid antibody titer elevation was unclear, although ultrasonography and thyroid scanning showed a decrease in thyroid volume. We diagnosed the patient with congenital hypothyroidism caused by thyroid dysgenesis. VWGS was diagnosed due to hypothyroidism, delayed bone age, and pseudoprecocious puberty. To the best of our knowledge, this is the first report of a prepubertal Korean boy with prolonged untreated congenital hypothyroidism complicated by VWGS in AS.
\end{abstract}

Keywords: Alport syndrome, Hypothyroidism, Van Wyk-Grumbach syndrome

\section{Introduction}

Alport syndrome (AS), first described by Cecil A. Alport in 1927, is a rare genetic disorder that causes progressive nephritis, which may ultimately lead to end-stage renal failure. ${ }^{1)}$ Some studies have examined the association between thyroid antibodies and hypothyroidism in people with AS, but most have been conducted among patients of European descent. Additionally, controversy remains regarding the relationship between hypothyroidism and thyroid antibodies in AS in adults. The relationship between thyroid disease and AS has rarely been reported, and there is no evidence regarding the relationship among Asians. ${ }^{2-5)}$

There are 2 types of hypothyroidism: congenital hypothyroidism $(\mathrm{CH})$ and acquired (autoimmune) hypothyroidism. The main cause of primary $\mathrm{CH}$ is thyroid dysgenesis, ${ }^{6}$ while autoimmune thyroiditis is the most common cause of acquired hypothyroidism. Antithyroid antibodies such as thyroid peroxidase antibody (anti-TPO Ab) and antithyroglobulin antibody (anti-Tg Ab) may be present. ${ }^{7)}$ Persistent hypothyroidism may lead to goiter, short stature, mental disabilities, constipation, neuropathy, and delayed sexual development. ${ }^{6)}$ However, children with long-standing hypothyroidism only rarely present with signs of precocious puberty, known as Van Wyk-Grumbach syndrome (VWGS). ${ }^{8)}$ VWGS is 
characterized by juvenile hypothyroidism, delayed bone age, and pseudoprecocious puberty. Very few cases of this condition have been reported in boys. ${ }^{9,10)}$ We report the case of a prepubertal Korean boy with VWGS and AS. The patient had $\mathrm{CH}$ and AS caused by thyroid dysgenesis without thyroid antibody elevation. Untreated hypothyroidism-induced VWGS was also present, along with other complications including short stature, obesity, and hyperlipidemia.

\section{Case report}

A boy 8 years and 4 months of age was admitted for short stature. He presented with a height of $109 \mathrm{~cm}(-3.5$ standard deviation score [SDS]), weight of $31.2 \mathrm{~kg}(1.08 \mathrm{SDS})$, and corresponding body mass index of $26.3 \mathrm{~kg} / \mathrm{m}^{2}$ (2.41 SDS). During genital examination, his testicular volume was Tanner stage II (6 mL bilaterally as measured using Prader orchidometer), while his pubic hair was classified as Tanner stage I. his stretched penile length was $4.5 \mathrm{~cm}$, which was within the normal range. ${ }^{11)}$ No goiter was found in his neck. Systolic blood pressure was $109 \mathrm{mmHg}$, diastolic blood pressure was 62 $\mathrm{mmHg}$, and heart rate was $75 \mathrm{bpm}$. No family history of organic disease, including kidney or thyroid disease, was reported. His newborn screening test results were all normal. The boy had previously been repeatedly admitted to our hospital at the age of 3 years and 2 months because of findings of gross hematuria and proteinuria. At the time, his height of $94.3 \mathrm{~cm}(-0.3 \mathrm{SDS})$, weight of $14.6 \mathrm{~kg}(0.12 \mathrm{SDS})$, and blood test results showed no specific findings, except for hyperlipidemia (total cholesterol [TC], $461 \mathrm{mg} / \mathrm{dL}$ ). AS was suspected on the basis of thin glomerular basement membrane and partial lamellation found in kidney biopsy, and regular follow-up was planned. However, the boy did not attend the regularly scheduled follow-up visits.

At age 8 years and 4 months, the boy was readmitted. His bone age, measured by the Greulich and Pyle method, was 6 years (Fig. 1). ${ }^{12)}$ Blood testing showed that his hemoglobin level was $11.0 \mathrm{~g} / \mathrm{dL}$, platelet level was $217 \times 103 / \mu \mathrm{L}$, and electrolyte levels were within the normal range. His TC level of $647 \mathrm{mg} /$ $\mathrm{dL}$ (normal range, $<170 \mathrm{mg} / \mathrm{dL}$ ), low-density lipoprotein cholesterol (LDL-C) level of $526 \mathrm{mg} / \mathrm{dL}$ (normal range, $<130$ $\mathrm{mg} / \mathrm{dL}$ ) and triglyceride level of $252 \mathrm{mg} / \mathrm{dL}$ (normal range,
$<75 \mathrm{mg} / \mathrm{dL}$ ) indicated hyperlipidemia. Other test results were as follows: luteinizing hormone (LH), $<0.07 \mathrm{mIU} / \mathrm{mL}$ (normal range, $0.02-0.3 \mathrm{mIU} / \mathrm{mL}$ ); follicle-stimulating hormone (FSH), $5.28 \mathrm{mIU} / \mathrm{mL}$ (normal range, $0.26-3.0 \mathrm{mIU} / \mathrm{mL}$ ); and testosterone, $13.35 \mathrm{ng} / \mathrm{dL}$ (normal range, $18-150 \mathrm{ng} / \mathrm{dL}$, Tanner stage II). His insulin-like growth factor-1 (IGF-1) level was $71.4 \mathrm{ng} / \mathrm{mL}$ (normal range at $8-9$ years, $64-345 \mathrm{ng} / \mathrm{mL}$ ) and IGF binding protein-3 level was 2,580 ng/mL (normal range at 8-9 years, 1,600-6,500 ng/mL) and cortisol $5.5 \mu \mathrm{g} / \mathrm{dL}$ (normal range, $3-21 \mu \mathrm{g} / \mathrm{dL}$ ). Blood urea nitrogen (BUN) level was 17.9 $\mathrm{mg} / \mathrm{dL}$ (normal range, $5-18 \mathrm{mg} / \mathrm{dL}$ ), and creatinine level was $0.6 \mathrm{mg} / \mathrm{dL}$ (normal range, $0.3-0.7 \mathrm{mg} / \mathrm{dL}$ ). Urinalysis showed gross hematuria and proteinuria and GFR was $127.69 \mathrm{~mL} / \mathrm{min}$. His thyroid function test results showed a free thyroxine level of $0.17 \mathrm{ng} / \mathrm{dL}$ (normal range, $0.64-1.72 \mathrm{ng} / \mathrm{dL}$ ) and a thyroidstimulating hormone (TSH) level of $871 \mathrm{mIU} / \mathrm{L}$ (normal range, 0.15-5.00 mIU/L), confirmatory of hypothyroidism.

Antithyroid antibody test results showed that his anti-Tg Ab level was $102.6 \mathrm{U} / \mathrm{mL}$ (normal range, $0-100 \mathrm{U} / \mathrm{mL}$ ), anti-TPO Ab level was $32 \mathrm{U} / \mathrm{mL}$ (normal range, $0-100 \mathrm{U} / \mathrm{mL}$ ), and TSHbinding immunoglobulin level was $<5 \mathrm{U} / \mathrm{L}$ (normal range, $0-9$ $\mathrm{U} / \mathrm{L}$ ). Thyroid ultrasonography was performed (Fig. 2), which showed decreased volume of both thyroid glands and the presence of multiple hypoechoic nodular lesions in both thyroid

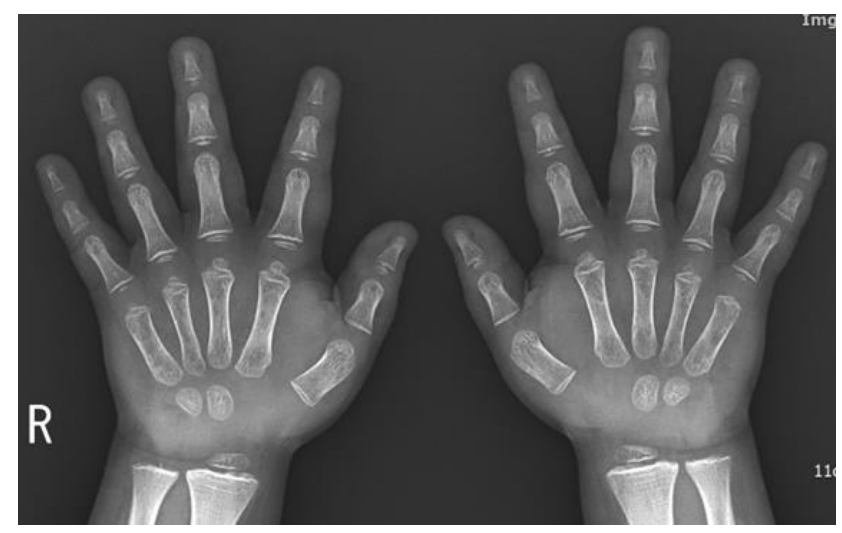

Fig. 1. Bone age radiograph obtained at the chronological age of 8 years and 4 months. The bone age was interpreted as 2 years 4 months using the Greulich and Pyle method.
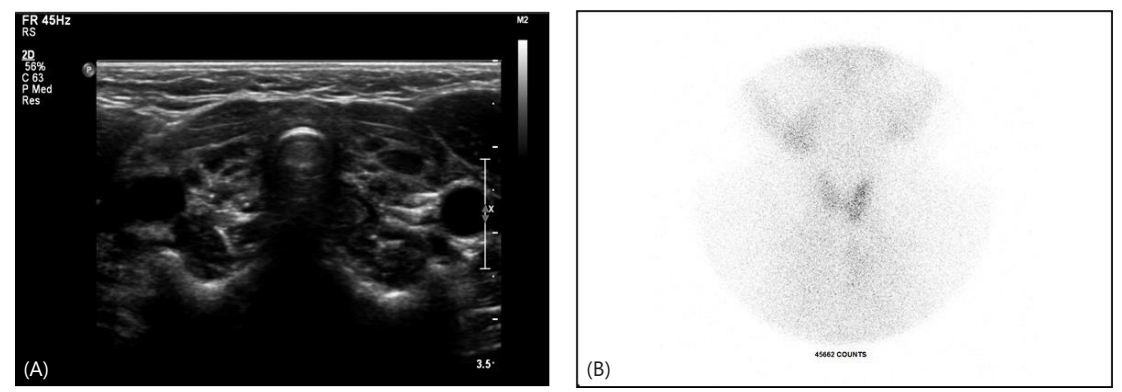

Fig. 2. (A) Thyroid ultrasonography showed decreased volume (thyroid volume, $2.5 \mathrm{~mL}$ ) of both thyroid glands. (B) A thyroid ${ }^{99 m}$ Tc scintigram also showed reduced size and uptake in both thyroid glands. Ectopic thyroid tissue was not detected. 
glands. Subsequent thyroid testing also confirmed decreased size and uptake in both thyroid glands. The patient was reassessed for the development of severe hypothyroidism, which was uncontrolled for a long time, and his Intelligence Quotient score was 64 , indicating a mild developmental disorder. Subsequently, a hearing test also confirmed sensorineural hearing loss in both ears. As the boy presented with hypothyroidism and hearing loss, a head computed tomography (CT) scan was performed to check for the possibility of Pendred syndrome. ${ }^{13)}$ The head CT scan revealed no inner ear malformation.

The boy underwent genetic testing for accurate diagnosis of AS. Sanger sequencing confirmed a de novo novel hemizygous variant, c.2600dupT [p. Gly869Arg], in exon 31 of the COL4A5 gene. The same variant was not observed on Sanger sequencing conducted for the mother.

The boy showed increasing testicle size in the pubertal form, ${ }^{14)}$ but his basal LH level was low $(<0.07 \mathrm{IU} / \mathrm{L})$ and his bone age was delayed. A gonadotropin-releasing hormone (GnRH) stimulation test was not performed.

Table 1. Follow-up of thyroid function test and antithyroid antibody titer

\begin{tabular}{lcccc}
\hline \multirow{2}{*}{ Variable (reference range) } & At diagnosis of & \multicolumn{4}{c}{ Follow-up (mo) } \\
\cline { 3 - 5 } & thyroiditis & 6 & 12 & 24 \\
\hline T3 (76-190 ng/dL) & 41 & 150 & 110 & 118 \\
FreeT4 $(0.64-1.72 \mathrm{ng} / \mathrm{dL})$ & 0.38 & 1.70 & 1.40 & 1.48 \\
TSH $(0.15-5.00 \mu \mathrm{lU} / \mathrm{mL})$ & 852 & 0.11 & 2.6 & 4.07 \\
Anti-Tg Ab $(0-100 \mathrm{U} / \mathrm{mL})$ & 102 & 49 & 31 & 49 \\
TPO antibody $(0-100 \mathrm{U} / \mathrm{mL})$ & 32 & $<15$ & 19 & 23 \\
TBII (0-9 U/L) & $<5$ & $<5$ & $<5$ & $<5$ \\
\hline
\end{tabular}

$\mathrm{TSH}$, thyroid-stimulating hormone; TPO, antithyroid peroxidase antibody; TBII, TSH-binding immunoglobulin.

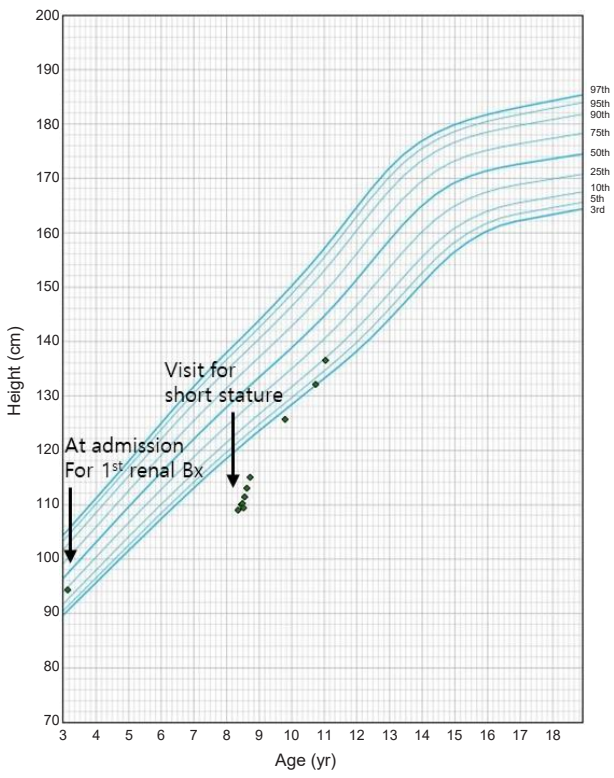

Upon completion of all tests, the boy was diagnosed with AS and prolonged hypothyroidism, which was considered as $\mathrm{CH}$ due to thyroid dysgenesis. VWGS was diagnosed based on hypothyroidism, delayed bone age, and pseudoprecocious puberty. To treat hypothyroidism, $3 \mu \mathrm{g} / \mathrm{kg} /$ day of levothyroxine sodium (Synthyroid) was administered. Blood tests were subsequently performed once per month until the patient's TSH level returned to normal. The administered dose was gradually increased and adjusted. Results of regular thyroid testing and thyroid autoantibody testing are summarized in Table 1. In regular growth rate checks after the start of drug administration, the boy showed increased growth as his thyroid function returned to normal and his weight decreased to within the normal range for his height (Fig. 3). Two years after starting treatment, normal thyroid function was maintained with administration of $0.1 \mathrm{mg}$ of Synthroid, his height increased to $136.5 \mathrm{~cm}(-1.0$ SDS), and his weight was $39.7 \mathrm{~kg}(0.39$ SDS). His bone age was 7 years 3 months, contrary to his chronological age of 10 years 9 months. Testicle size decreased from $6 \mathrm{~mL}$ to $5 \mathrm{~mL}$. The boy has been taking ACE inhibitors since the age of 8 years and 5 months. In a regularly scheduled urinalysis for AS, intermittent proteinuria and microscopic hematuria were found, but kidney function test results showed that his BUN $(12.5 \mathrm{mg} / \mathrm{dL})$ and creatine $(0.24 \mathrm{mg} / \mathrm{dL})$ levels were maintained at normal levels.

\section{Discussion}

AS is a rare genetic disorder that causes progressive nephritis and is more common among men. Known symptoms (other than kidney-related) include sensorineural hearing loss and corneal anomalies. ${ }^{1)}$ An abnormality in the COL4A5 gene

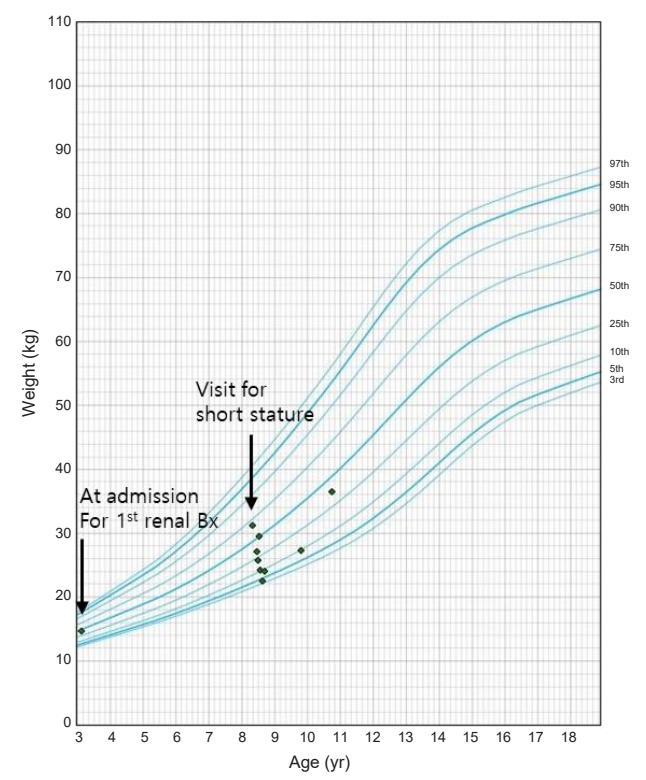

Fig. 3. Growth curves showing that the patient had achieved recovery of normal growth rate following the start of thyroid hormone therapy. Bx, biopsy. 
associated with the $\mathrm{X}$ chromosome has been identified in $85 \%$ of AS cases. ${ }^{15)}$ The boy in the present case was diagnosed with AS based on the results of a kidney biopsy performed due to hearing loss and repeated findings of gross hematuria and proteinuria. Targeted exome sequencing revealed a de novo novel hemizygous variant c.2600dupT [p. Gly869Arg] in exon 31 of the COL4A5 mutation. An association between the COL4A5 gene and thyroid disease has not yet been identified.

There are prior reports of thyroid dysfunction in white subjects with a confirmed diagnosis of AS. ${ }^{2,3)}$ De Marchi and Cecchin $^{2)}$ measured antithyroid antibody levels in the blood of patients with AS in 3 households. Antithyroid antibody levels were compared to a healthy control group, a dialysis patient group, and an AS group. The AS group showed significantly higher antithyroid antibody levels than the other 2 groups. Miyoshi et al. ${ }^{3)}$ reported that antithyroid antibody levels were higher in patients with AS than in a healthy control group. However, Savige et al. ${ }^{4)}$ measured antithyroid and antiadrenal autoantibodies in patients with antiglomerular basement membrane disease, thin basement membrane disease, and AS. No patient with AS had antithyroid antibodies. They concluded that antithyroid and antiadrenal antibodies are not associated with AS.

There remains controversy about the relationship between hypothyroidism and thyroid antibodies in AS. In our case, the antithyroid antibody levels were within the normal range. However, ultrasonography showed decreased volume of both thyroid glands, which was confirmed by the decreased size and uptake of both thyroid glands. No ectopic thyroid gland was present. Ultrasonography may reveal diffuse reduction in thyroid echogenicity in autoimmune thyroid disease. ${ }^{16)}$ Thyroid dysgenesis includes athyreosis, that is, an "empty" thyroid area with ectopic thyroid tissue and thyroid hypoplasia (global hypoplasia and thyroid hemigenesis). ${ }^{6}$ Thus, we hypothesized that the boy's hypothyroidism was caused by thyroid dysgenesis. However, genetic testing related to thyroid dysgenesis is necessary to establish a definitive diagnosis.

In the present case, the boy had growth deficiency, obesity, and bone age delay due to prolonged untreated hypothyroidism. However, there was a pubertal increase in testicle size, ${ }^{17)}$ which was not accompanied by any other obvious signs of virilization. His basal LH level was very low $(<0.07 \mathrm{mIU} / \mathrm{mL})$ and his bone age was delayed. A GnRH stimulation test was not performed. Pasternak et al. ${ }^{14)}$ suggested that a single basal LH cutoff value of $0.1 \mathrm{IU} / \mathrm{L}$ had a specificity of $94.7 \%$ and a sensitivity of $64 \%$. Single basal LH measurement may be adequate to confirm but not to refute the presence of central precocious puberty (CPP). When clinically indicated as evidenced by progression of pubertal signs, accelerated growth, and bone age advancement, a GnRH stimulation test should be applied for the diagnosis of $\mathrm{CPP}{ }^{14)}$ VWGS is associated with delayed bone age, which differs from CPP, which is associated with growth acceleration and bone age advancement. This difference can be explained based on thyroid hormone function. Thyroid hormone-mediated bone maturation includes growth hormone gene expression and IGF-system regulation. Sex steroids are responsible for the pubertal growth spurt and epiphyseal fusion. ${ }^{18)}$ Our case was diagnosed with VWGS based on hypothyroidism, delayed bone age, and pseudoprecocious puberty. Two years after thyroxine replacement, his testicle size decreased from 6 $\mathrm{mL}$ to $5 \mathrm{~mL}$ and his bone age was also delayed. However, his testicle size was normal for his pubertal level, which limits the diagnosis of VWGS. If testicle size no longer decreases during thyroid hormone treatment, GnRH stimulation test should be performed.

Hypothyroidism, if left untreated for a long period, may lead to delayed puberty. In rare cases, it may be accompanied by precocious puberty, such as VWGS. ${ }^{8,11,12,19)}$ Most cases of VWGS have been reported in prepubertal girls, while male cases are rarer and present with testicular enlargement. ${ }^{9,10)}$ There are several hypotheses to explain these paradoxical symptoms. Both gonadotropins and TSH are glycoproteins; their overlap at the hormonal level is associated with the lack of specificity at the hypothalamic level. ${ }^{9)}$ Moreover, an increase in TSH levels induced by the thyrotropin-releasing hormone may act as an agonist of FSH receptors (FSH-R) without a simultaneous stimulation of LH receptors. TSH continuously stimulates FSH-R to induce pubertal changes. ${ }^{9,10)}$ In male VWGS patients, testicle volume increases without any virilization due to FSH-R continuous activation and Sertoli cell proliferation. ${ }^{19)}$

Hyperlipidemia is also thought to be caused by hypothyroidism. Hypothyroidism can have an important effect on lipid profiles. Thyroid hormones increase 3-hydroxy-3 methylglutaryl-coenzyme A reductase activity, which upregulates LDL receptors and can also influence high-density lipoprotein metabolism. ${ }^{20)}$ Thus, patients with hypothyroidism have increased levels of TC and LDL-C. Our patient's hyperlipidemia improved to the normal range after 2 months of thyroxine treatment.

In conclusion, we report the first case of a Korean boy with VWGS induced by prolonged untreated CH in AS. VWGS was also present, in addition to general symptoms such as short stature, obesity, and hyperlipidemia.

\section{Ethical statement}

This case was approved by the Institutional Review Board of Kyungpook National University Chilgok Hospital (approval number: 2019-04-007). Informed consent was obtained from the patient's parents.

\section{Conflict of interest}

No potential conflict of interest relevant to this article was reported.

\section{References}

1. Kashtan CE. Alport syndrome and thin glomerular base- 
ment membrane disease. J Am Soc Nephrol 1998;9:173650 .

2. De Marchi S, Cecchin E. Asymptomatic autoimmune thyroiditis and thyroid dysfunction in Alport's syndrome. A report of three families. Nephrol Dial Transplant 1991;6:7985.

3. Miyoshi K, Suzuki M, Ohno F, Yamano T, Yagi F. Antithyroid antibodies in Alport's syndrome. Lancet 1975;2:480-2.

4. Savige JA, Branley P, Neeson P, Holdsworth S, Thurlow P. Antithyroid and antiadrenal autoantibodies in antiglomerular basement membrane disease, thin basement membrane disease and Alport syndrome. Pathology 1998;30:30-3.

5. Lambert M, Pirson Y, De Nayer P, van Ypersele de Strihou C. No association between Alport's syndrome and antithyroid antibodies. Nephrol Dial Transplant 1992;7:1082-4.

6. Kollati Y, Ambati RR, Reddy PN, Kumar NSS, Patel RK, Dirisala VR. Congenital hypothyroidism: facts, facets \& therapy. Curr Pharm Des 2017;23:2308-13.

7. Caturegli P, De Remigis A, Rose NR. Hashimoto thyroiditis: clinical and diagnostic criteria. Autoimmun Rev 2014; 13:391-7.

8. Van Wyk JJ, Grumbach MM. Syndrome of precocious menstruation and galactorrhea in juvenile hypothyroidism: an example of hormonal overlap in pituitary feedback. J Pediatrics 1960;57:416-35.

9. Zhang S, Yang J, Zheng R, Jiang L, Wei Y, Liu G. VanWykGrumbach syndrome in a male pediatric patient: a rare case report and literature review. Exp Ther Med 2017;13:1151-4.

10. Omran A, Peng J, Shrestha B, Ashhab MU, Yin F. Male child with Van Wyk-Grumbach's syndrome and other complications of long-standing primary hypothyroidism: a case report. Case Rep Pediatr 2012;2012:352751.
11. Hatipoğlu N, Kurtoğlu S. Micropenis: etiology, diagnosis and treatment approaches. J Clin Res Pediatr Endocrinol 2013;5:217-23.

12. Greulich WW, Pyle SI. Radiographic atlas of skeletal development of the hand and wrist. 2nd ed. Stanford (CA): Stanford University Press, 1999.

13. Glaser B. Pendred syndrome. Pediatr Endocrinol Rev 2003;1 Suppl 2:199-204.

14. Pasternak Y, Friger M, Loewenthal N, Haim A, Hershkovitz E. The utility of basal serum LH in prediction of central precocious puberty in girls. Eur J Endocrinol 2012;166:2959.

15. Bekheirnia MR, Reed B, Gregory MC, McFann K, Shamshirsaz AA, Masoumi A, et al. Genotype-phenotype correlation in X-linked Alport syndrome. J Am Soc Nephrol 2010;21:876-83.

16. Vlachopapadopoulou E, Thomas D, Karachaliou F, Chatzimarkou F, Memalai L, Vakaki M, et al. Evolution of sonographic appearance of the thyroid gland in children with Hashimoto's thyroiditis. J Pediatr Endocrinol Metab 2009;22:339-44.

17. Biro FM, Lucky AW, Huster GA, Morrison JA. Pubertal staging in boys. J Pediatr 1995;127:100-2.

18. Robson H, Siebler T, Shalet SM, Williams GR. Interactions between GH, IGF-I, glucocorticoids, and thyroid hormones during skeletal growth. Pediatr Res 2002;52:137-47.

19. Reddy P, Tiwari K, Kulkarni A, Parikh K, Khubchandani R. Van Wyk Grumbach syndrome: a rare consequence of hypothyroidism. Indian J Pediatr 2018;85:1028-30.

20. Duntas LH, Brenta G. The effect of thyroid disorders on lipid levels and metabolism. Med Clin North Am 2012;96:269-81. 